\title{
Topographical measurement of the attachments of the central band of the interosseous membrane on interosseous crests of the radius and ulna
}

\author{
Suk-Hwan Jang ${ }^{1}$, Kyung-Whan Kim ${ }^{2}$, Hyo Seok Jang ${ }^{3}$, Yeong-Seok Kim ${ }^{4}$, Hojin Kim ${ }^{3}$, Youngbok Kim ${ }^{3}$ \\ ${ }^{1}$ Department of Orthopedic Surgery, Seoul Paik Hospital, Inje University, Seoul, Korea \\ ${ }^{2}$ Department of Orthopedic Surgery, Zion Hospital, Busan, Korea \\ ${ }^{3}$ Department of Orthopedic Surgery, Haeundae Paik Hospital, Inje University, Busan, Korea \\ ${ }^{4}$ Department of Anatomy, Inje University, Busan, Korea
}

Background: To suggest a reasonable isometric point based on the anatomical consistency of interosseous membrane (IOM) attachment in association with topographic characteristics of the interosseous crests, the footprints of the central band (CB) of the IOM on the radial and ulnar interosseous crests (RIC and UIC) were measured.

Methods: We measured the distance from the CB footprints from each apex of both interosseous crests in 14 cadavers and the angles between the forearm axis of rotation (AOR) and the distal slopes of the RIC and UIC in 33 volunteers.

Results: The CB footprints lay on the downslope of both interosseous crests with its upper margin on average 3-mm proximal from the RIC's apex consistently in the radial length, showing normality ( $>0.05)$, and on average 16-mm distal from the UIC's apex on the ulna without satisfying normality ( $\mathrm{p}<0.05)$. The average angle between the UIC's distal slope and the AOR was $1.3^{\circ}$, and the RIC's distal slope to the AOR was $14.0^{\circ}$, satisfying the normality tests ( $p>0.05$ ), and there was no side-to-side difference in both forearms $(p<0.05)$.

Conclusions: The CB attached to the downslope just distal to the RIC's apex constrains the radius to the UIC that coincides with the AOR of the forearm circumduction, maintaining itself both isometrically and isotonically.

Keywords: Interosseous crest; Radius; Ulna; Interosseous membrane; Central band

\section{INTRODUCTION}

The interosseous membrane (IOM) of the forearm is a complex anatomical structure in the form of a strong band connecting the radius and ulna. By constraining the radius relative to the ulna, the IOM acts to maintain a constant radioulnar relationship during rotational movements of the forearm and to transfer and distribute axial loads arising from the hand from the radius to the ulna. The IOM consists of the following five distinctive ligaments: the central band (CB), distal oblique bundle, proximal oblique cord, dorsal oblique accessory cord, and accessory band [1]. Of these, the $\mathrm{CB}$, which originates from the proximal radius and inserts into the distal ulna, is a strong, stable structure responsible for the major functions of the IOM $[2,3]$. The CB is

Received: August 18, 2021 Revised: October 18, 2021 Accepted: October 26, 2021

Correspondence to: Youngbok Kim

Department of Orthopedic Surgery, Haeundae Paik Hospital, Inje University, 875 Haeun-daero, Haeundae-gu, Busan 48108, Korea

Tel: +82-51-781-5704, Fax: +82-51-797-0991, E-mail: H00151@paik.ac.kr, ORCID: https://orcid.org/0000-0002-7966-1092

Financial support: None.

Conflict of interest: None.

Copyright@ 2021 Korean Shoulder and Elbow Society.

This is an Open Access article distributed under the terms of the Creative Commons Attribution Non-Commercial License (http://creativecommons.org/licenses/by-nc/4.0/) which permits unrestricted non-commercial use, distribution, and reproduction in any medium, provided the original work is properly cited. 
known to maintain a constant length and tension regardless of forearm rotation $[1,4]$. When comparing the forearm rotation to a simple hinge motion, the radius and ulna may correspond to each leaf of the hinge; the $\mathrm{CB}$ may be reduced to the pin joining the leaves; and the radial interosseous crest (RIC) and ulnar interosseous crest (UIC) act as the knuckles holding the pin. However, forearm rotation is not a simple opening and closing of the radio-ulnar hinge complex but instead a circumduction in which the radius traces a cone reciprocating around the ulnar shaft $[5,6]$. In the setting of such conical motion geometry, we hypothesized that the attachment points of the $\mathrm{CB}$ should be uniformly located in accordance with the shapes of the RIC and UIC because the geometric elements of the interosseous crests should maintain the CB both isometrically and isotonically regardless of the degree of the forearm rotation. Such control elements should include a solid axis of rotation (AOR) and topographical aspects of ligament attachment that dynamically tune the tension and relaxation portions depending upon the degree of forearm rotation. Thus, in this study, the authors performed anatomical measurements on cadaveric specimens and volunteers to investigate a coherent structure to the AOR in the forearm and the spatial consistency of the attachments of the $\mathrm{CB}$.

\section{METHODS}

This study was approved by the Institutional Review Board of Inje University (IRB No. INJE 2017-03-014-002). Informed consent from patients was waived, and informed consent from the participant in the figure was obtained for publication of the photographs. For the cadaveric investigation, 14 (10 male and four female) fresh frozen upper arm specimens (mean age at death, 72 years; range, 57-83 years) were obtained; after elbow and wrist joint disarticulation, gross observation and radiography were used to exclude any specimens showing lesions related to degenerative change or old trauma as well as specimens where the $\mathrm{CB}$ could not be clearly distinguished due to the IOM being too thin. The triangular fibrocartilage complex was retained at the wrist joint to preserve the distal radioulnar joint, and the annular ligament was retained at the elbow joint to preserve the proximal radioulnar joint. An osteoligamentous forearm complex was prepared by removing all soft tissues from the forearm, except for the pronator quadratus, pronator teres, and IOM (Fig. 1). The CB was identified within the IOM using the backlit method [1,7] with a surgical light; the four corners of the $\mathrm{CB}$ on the RIC and UIC were marked and drilled with a 1.0-mm K-wire to insert $1.0-\mathrm{mm}$-thick lead markers for the radiographs. The backlit method has a disadvantage in that the selection range of the IOM may vary somewhat depending upon the illuminance and distance from the light source, but it is regarded as a feasible method by which to distinguish the thickest part of the IOM. We tried to distinguish the $\mathrm{CB}$ at around $50 \mathrm{~cm}$ from the regular operating room light by referring to the methods of existing researchers [1]. In addition, the isometricity was confirmed by measuring the length changes of the proximal and distal marginal fibers of the selected portion of the IOM in the end-pronation, neutral, and end-supination positions of the forearm. We performed the same measurements on both forearms for data-collection purposes. Still, we chose unilateral data from (six right forearms and eight left forearms) for analysis by random selection to avoid duplication of the data because the measurement values from both forearms tended to be similar for each individual, and the number of cadavers was small.

For the volunteer investigation, 66 forearms from 33 volunteers, including 16 men and 17 women (mean age, 28 years;

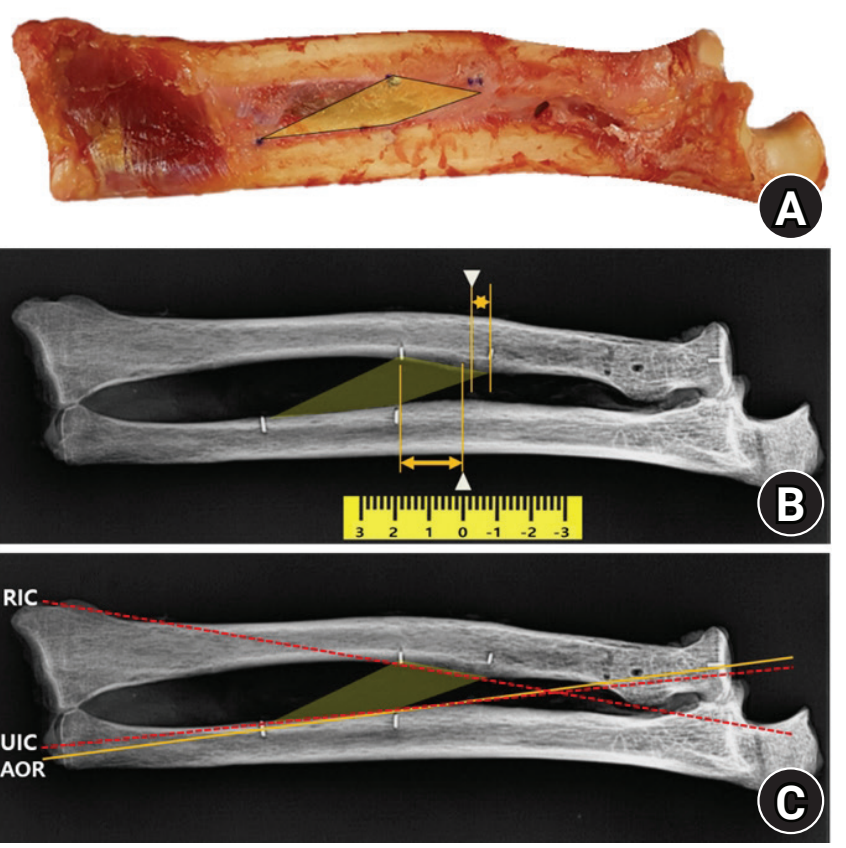

Fig. 1. Measurements from radiographs (palm-up oblique view). When a forearm specimen that has been processed to a forearm osteoligamentous complex is placed naturally on the cassette (A), the ulna becomes externally rotated and the radius becomes internally rotated by itself, aligning both interosseous crests in a single plane (B). The border of the central band (CB) was drawn by joining up the lead markers that had previously been inserted at the four corners of the CB. The distances were measured from each apex (arrowheads) of the interosseous crests on the radius and ulna to the proximal margin of the $\mathrm{CB}$ to ascertain the positions of the $\mathrm{CB}$ footprints relative to the ulnar interosseous crest (UIC) and radial interosseous crest (RIC) (double arrows). In addition, the angles are measured between the axis of rotation (AOR) of the forearm (solid line) and the lines extrapolated from the downslopes of the RIC and UIC (dotted lines) (C). 
range, 23-44 years), were involved. Since the interosseous crests of both bones project differently, i.e., the RIC anteromedially and the UIC anterolaterally, it is necessary to align them in a single plane. In cadaveric specimens, this forearm configuration could be set simply by placing the osteoligamentous forearm complex naturally on the cassette without manipulation (Fig. 1). In contrast, volunteers rotated their entire forearm externally to turn the ulna externally and rotated only the hand internally to turn the radius internally. We labeled this radiograph a "palm-up oblique view" because only the palm faced upward, yielding a conventional external oblique view of the forearm (Fig. 2). Data from both forearms were used for analysis to investigate the symmetry of the left and right forearms and the consistency of repeated measurements through the measurements of both sides. All statistical analyses were conducted by a statistics expert using the IBM SPSS ver. 21.0 (IBM Corp., Armonk, NY, USA); results with $\mathrm{p}<0.05$ were considered to be statistically significant.

\section{Anatomical Measurements and Confirmation of CB Isometricity}

With the cadaveric forearms in end-supination, the distances between the four corners of the $\mathrm{CB}$ were measured to discern the lengths of the radial footprint, ulnar footprint, proximal margin, and distal margin. The proximal and distal margins were measured repeatedly in the neutral position and in end-pronation to verify whether the $\mathrm{CB}$ maintained isometricity regardless of forearm rotation. All measurements were performed with an accuracy of $0.1 \mathrm{~mm}$ by a single orthopedic specialist using Vernier calipers (accuracy, $0.05 \mathrm{~mm}$; Mitutoyo, Kanagawa, Japan); in neutral and end-pronation positions, a divider was used to measure the distances between the points when the measurement point was inaccessible with the caliper. The Shapiro-Wilk test was performed to confirm the normality of the measurements.

\section{Location of the Footprints of the $\mathrm{CB}$ and Each Apex of the RIC and UIC}

This analysis sought to examine how the geometry of the interos-
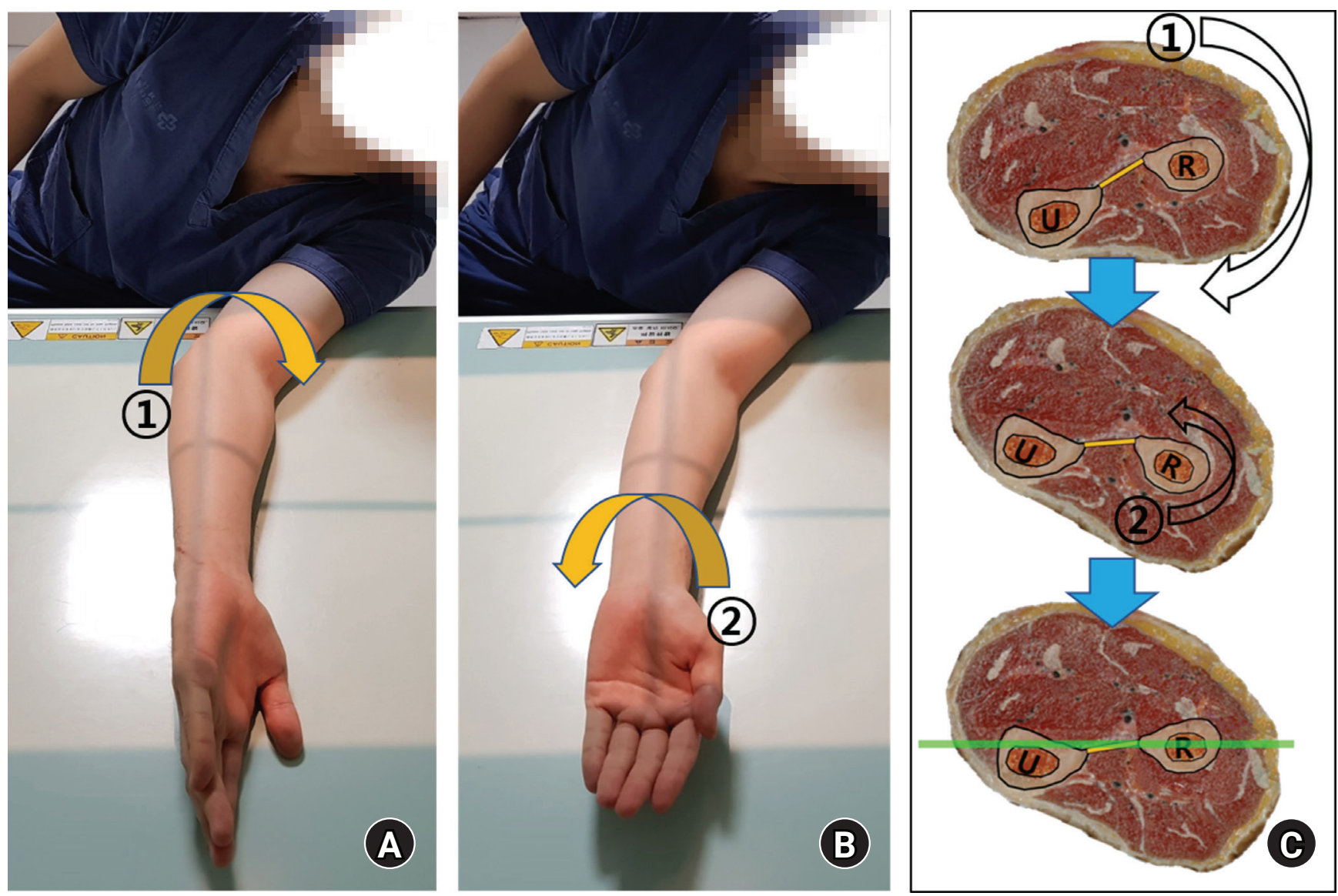

Fig. 2. Palm-up oblique view radiography method. The patient is seated, initially placing the forearm on the cassette in the same position as a conventional $45^{\circ}$ external oblique view (A), then asked to rotate only the forearm internally until the palm faces up (B). This method aligns the interosseous crests of both bones with different projections into the same plane for imaging (C). U: ulna, R: radius. 
seous crests of both bones could associate with the actions of the $\mathrm{CB}$. On the palm-up oblique view radiographs from the volunteers, each apex of the RIC and UIC was defined as 0; the direction proximal and distal to the apex was defined as positive $(+)$ and negative (-), respectively. The distance from each apex to the upper end of the CB footprint was measured on the RIC and UIC. A normality test was performed for the measurements. The highest point in the outline of each interosseous crest when viewed from the front was defined as the apex, and the slope extending from the apex to the distal part was defined as the downslope.

\section{Angular Configuration of the RIC and UIC to the Forearm AOR}

On the palm-up oblique view radiographs from the volunteers, we drew a line from the center of the articular surface of the radial head to the styloid process of the ulna to indicate the AOR $[5,8]$ of the forearm, then measured the angle with lines drawn along the downslopes of the interosseous crests of the radius and ulna. The purpose of this measurement was to examine the coherent anatomy related to the AOR in the conical track of the forearm rotation [6,9]. The Shapiro-Wilk test was performed to confirm the normality of the measurements.

\section{RESULTS}

\section{Anatomical Measurements and Confirmation of CB Isometricity}

The mean lengths of the radial footprint and ulnar footprint of the $\mathrm{CB}$ measured in end-supination were $27.6 \pm 4.3 \mathrm{~mm}$ (range, $22-43 \mathrm{~mm}$ ) and $32.8 \pm 6.6 \mathrm{~mm}$ (range, $25-50 \mathrm{~mm}$ ), respectively. The mean width in the middle portion measured perpendicular to its fibers was $18.5 \pm 3.5 \mathrm{~mm}$ (range, $11-26 \mathrm{~mm}$ ). When measurements of the length of the $\mathrm{CB}$ were repeated in different forearm rotations at proximal and distal margins, the results were as follows: proximal margin, $41.7 \pm 6.4 \mathrm{~mm}$ and distal margin, $45.0 \pm 5.5 \mathrm{~mm}$ in end-supination; proximal margin, $41.7 \pm 6.4$ $\mathrm{mm}$ and distal margin, $45.1 \pm 5.3 \mathrm{~mm}$ in neutral rotation; and proximal margin, $41.7 \pm 6.5 \mathrm{~mm}$ and distal margin, $45.0 \pm 4.9 \mathrm{~mm}$ in end-pronation. No statistically significant differences were found in CB length at varying degrees of forearm rotation (repeated measures analysis of variance test, $\mathrm{p}=1.000$ and $\mathrm{p}=0.923$, respectively), confirming the isometricity of the $\mathrm{CB}$ selected by our method (Fig. 3).

\section{Location of the Footprints of the $\mathrm{CB}$ and Each Apex of the RIC and UIC}

On the RIC, the proximal margin of the CB inserted into a point $2.9 \pm 1.1 \mathrm{~mm}$ proximal $(+)$ to the RIC's apex; on the ulna, the proximal margin of the CB inserted into a point $14.7 \pm 6.5 \mathrm{~mm}$ distal (-) to the UIC's apex. The measured values for the radial footprints satisfied the normality test, but those of the ulnar footprints did not (Shapiro-Wilk test, $\mathrm{p}=0.13$ and $\mathrm{p}=0.02$, respectively) (Fig. 4). Since the proximal margin of the CB attaches adjacent to the apices of the RIC and UIC on both sides, the rest of the footprints of the $\mathrm{CB}$ lie distal to these points along the downslopes of both interosseous crests. When the location of the
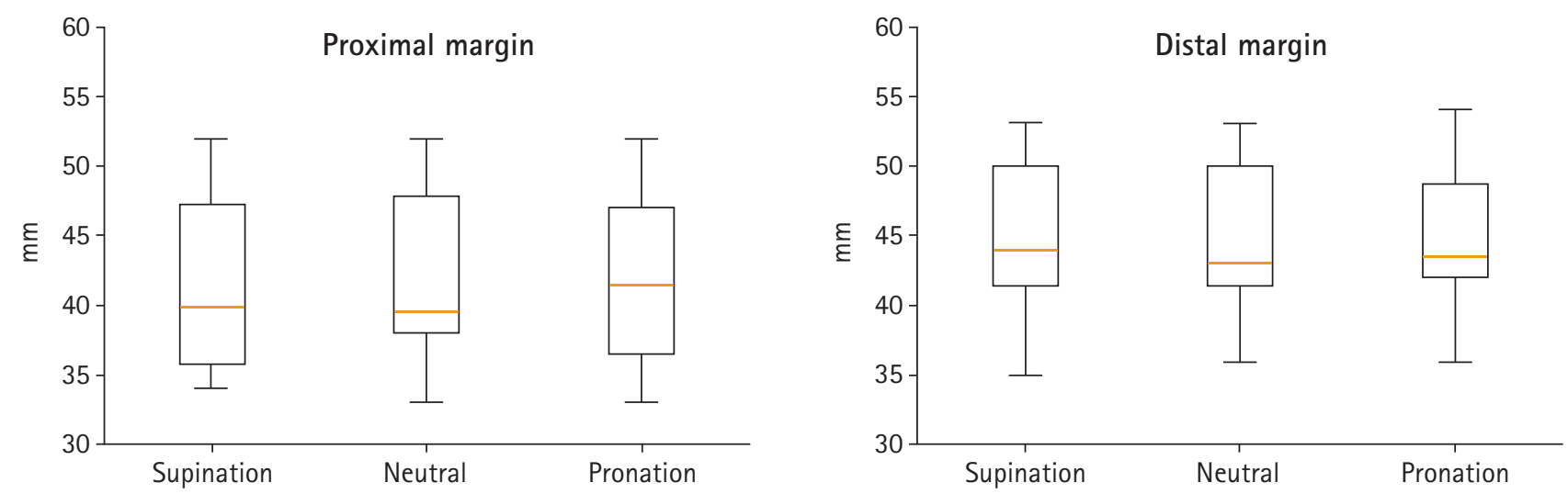

\begin{tabular}{ccccc}
\hline Variable & Supination & Neutral & Pronation & p-value \\
\hline Proximal margin & $41.71 \pm 6.43$ & $41.71 \pm 6.46$ & $41.71 \pm 6.51$ & 1.000 \\
Distal margin & $45.07 \pm 5.51$ & $45.07 \pm 5.51$ & $45.00 \pm 4.87$ & 0.923 \\
\hline
\end{tabular}

Fig. 3. Changes in central band (CB) length based on the extent of forearm rotation. No statistically significant differences were found in the $\mathrm{CB}$ length measured in end-supination, neutral rotation, or end-pronation (repeated measures analysis of variance test, $p>0.05$ ), confirming $\mathrm{CB}$ isometricity. This demonstrates that the portion selected for measurements in the present study is the major bundle of isometric fibers in the CB. 

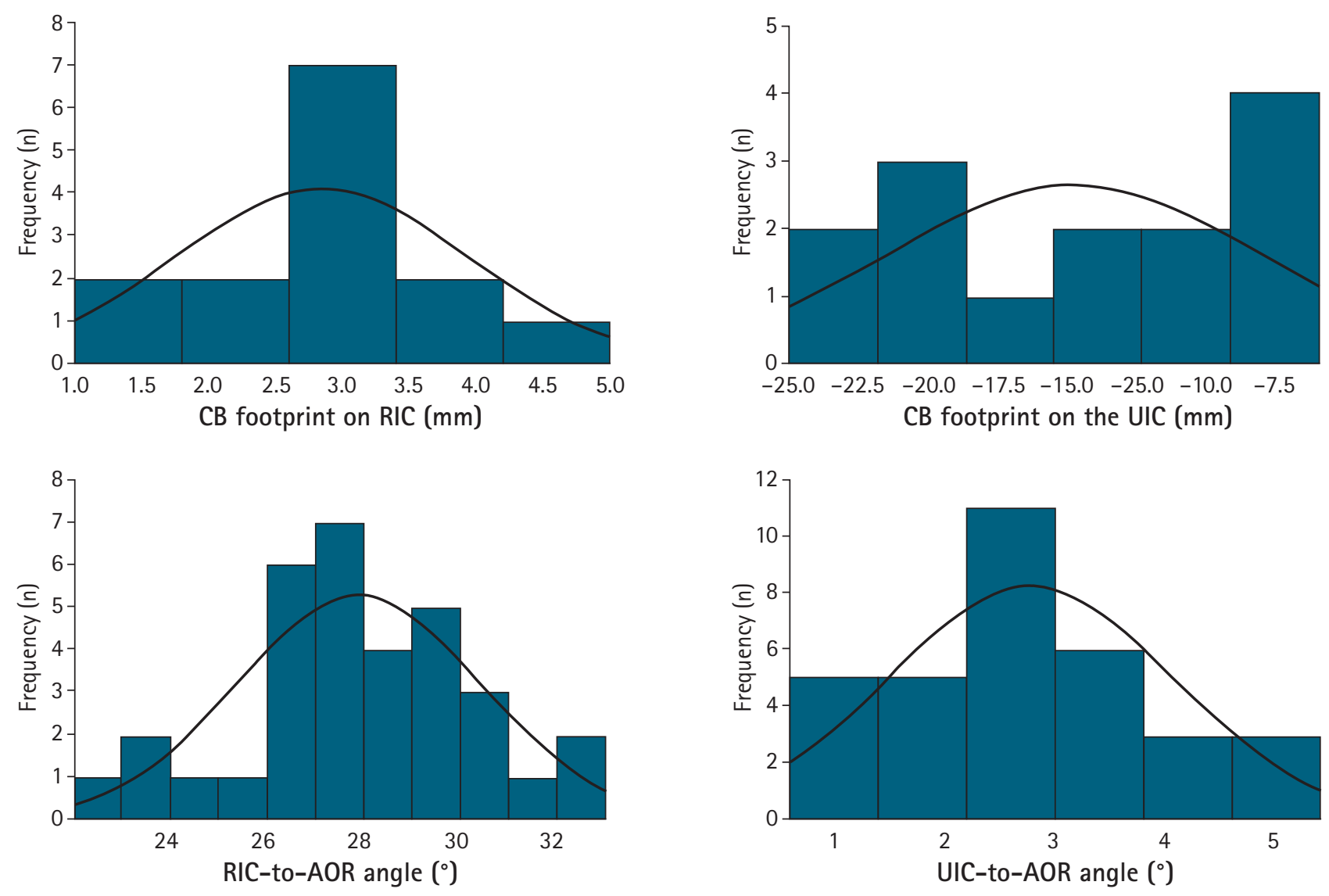

Fig. 4. Location of the central band (CB) footprints and the angular configurations of the forearm axis of rotation (AOR) to the interosseous crest (RIC) and ulnar interosseous crest (UIC). The distance of proximal margin of the CB footprint is approximately $3 \mathrm{~mm}$ proximal to the RIC's apex on the radius with normality (Shapiro-Wilk test, $\mathrm{p}>0.05$ ) and $15 \mathrm{~mm}$ distal to the UIC's apex on the ulna with no normality (Shapiro-Wilk test, $\mathrm{p}<0.05)$. This measurement indicates that the $\mathrm{CB}$ attaches along the downslopes of both interosseous crests distally to each apex. The UIC coincides with the forearm AOR, and the RIC is at about $15^{\circ}$ to this axis with normality (Shapiro-Wilk test, $\mathrm{p}>0.05$ ). n: the number of the specimens or volunteers in the measurement interval.

apex of each interosseous crest was measured by defining the radial length from the radial head top to the radial styloid process and the ulnar length from the olecranon tip to the ulnar styloid process, respectively, the RIC's apex was on average $62.2 \%$ (mean, $151 \mathrm{~mm} / 242 \mathrm{~mm}$ ) and the UIC's apex was on average $51.8 \%$ (mean, $135 \mathrm{~mm} / 262 \mathrm{~mm}$ ) from the distal ends of both bones. The measurements for the location of the RIC's apex satisfied normality (Shapiro-Wilk test, $\mathrm{p}=0.120$ ), but those of the UIC's apex did not (Shapiro-Wilk test, $\mathrm{p}=0.007$ ). There was no statistical difference in the position of each apex on the radial and ulnar lengths in the volunteers (paired t-test, $\mathrm{p}=0.09$ ).

\section{Angular Configuration of the RIC and UIC to the Forearm AOR}

After drawing lines to overlap the downslopes of the RIC and UIC (Fig. 1), we measured the angles of these lines with the forearm AOR. On both sides of the forearm, the mean angles of the
RIC to AOR and UIC to AOR were $14.0^{\circ} \pm 1.3^{\circ}$ and $1.4^{\circ} \pm 0.65^{\circ}$, respectively. Both these measurements satisfied the normality test (Shapiro-Wilk test, $\mathrm{p}=0.24$ and $\mathrm{p}=0.92$, respectively) (Fig. 4). The line extrapolated from the downslope of the UIC was almost identical to the forearm AOR; this means that the ulnar footprint of the $\mathrm{CB}$ inserts precisely along the forearm AOR [8], holding the radius as a firm center of the rotation during forearm circumduction.

In order to test the reliability of repeated measurements for this angular configuration and symmetry between both forearms, we performed an analysis by separating the left and right forearms. The mean angles of the RIC and UIC with the AOR on each side were as follows: right, $14.4 \pm 1.3$ and left, $13.9 \pm 1.3$ on the RIC and right, $1.4 \pm 0.6$ and left, $1.4 \pm 0.6$ on the UIC, respectively. There was no significant statistical difference between sides (paired t-test, $\mathrm{p}=0.19$ and $\mathrm{p}=0.08$, respectively). 


\section{DISCUSSION}

Although there are some different opinions regarding the anatomical structure and components of the IOM in the literature, there seems to be no disagreement that the $\mathrm{CB}$ is the most functional element as a stout and constant structure [8,10-13]. The $\mathrm{CB}$ works as a restraint on the radius from proximal migration in cooperation with the radial head and works as a load transmitter between the radius and ulna to redistribute load [14-16]. Several authors have demonstrated that the $\mathrm{CB}$ is an isometric ligament of the IOM with no change in length or tension occurring during forearm rotation $[4,8,11,15,17]$. In contrast to the anatomical and functional understanding of the $\mathrm{CB}$, there has been little explanation offered about how this band-shaped structure maintains its isotonicity and isometricity while twisting and fanning during the rotation of the forearm. The authors hypothesized that the morphology of the RIC and UIC would perform a specific function for the $\mathrm{CB}$ to restrain the two bones of the forearm without restricting the rotational motion of the forearm in a dynamic environment. This assumption implies a secondary hypothesis of that the RIC and UIC should have predictable shapes and that the attachments of the $\mathrm{CB}$ will be distributed with regularity at specific sites in association with such a topographic element. Regarding the attachment point of the $\mathrm{CB}$ on the radius and ulna, several researchers have described it as a ratio to the radial and ulnar length or the distance from adjacent structures. Skahen et al. [15] reported that the CB begins $7.7-\mathrm{cm}$ distal to the articular surface of the radial head, inserts $13.7-\mathrm{cm}$ distal to the tip of the olecranon, and is aligned at an angle of $21^{\circ}$ from the proximal radius toward the distal ulna. Marcotte and Osterman [3] reported that the ulnar insertion is located at a distance of $33 \%$ of the ulnar length from the styloid process, while the radial insertion is located $60 \%$ of the radial length from the radial styloid process. Noda et al. [1] investigated the location of the $\mathrm{CB}$ insertion point using the distance from the distal part as a proportion of the whole length and reported that the radial insertion is located $53 \% \pm 4 \%$ and $64 \% \pm 5 \%$ from the distal and proximal ends, respectively, whereas the ulnar insertion is located $29 \% \pm 4 \%$ and $44 \% \pm 5 \%$ from the distal and proximal ends, respectively. Currently, in clinical practice, these indicators provide rough guidelines for reproducing the isometric point of the $\mathrm{CB}$. Still, due to the lack of explanation for the anatomical or biomechanical necessity of such attachment characteristics, these methodologies might be interpreted differently depending on race and individual.

Forearm motion, which is based on rotation of the radius around the ulna, can be simplified as a hinge or bookbinding [9], wherein the two bones are the leaves, the $\mathrm{CB}$ is the pin, and the RIC and UIC are the knuckles. In this simplified model, we make the biomechanical assumption that the $\mathrm{CB}$ will show no great change in length throughout the whole range of forearm rotation $[1,4,18]$, while constraining the radius stably and with constant degrees of freedom relative to the ulna. This enables the hypothesis that the bony geometry might play a role in controlling the $\mathrm{CB}$ during forearm rotation; hence, we focused on the topography of the RIC and UIC, where the CB footprints are located.

Mori [11] described the forearm AOR as coinciding with the interosseous border of the ulna (UIC), and Hollister et al. [8] showed that all fibers of the IOM crossed the forearm AOR near insertion in the ulna. Their results suggest that the ulnar attachment of the IOM sits consistently aligned in line with the forearm AOR by attaching to the UIC, which is a solid and invariant bony border. We reconfirmed that the forearm AOR and distal slope of the UIC coincided within $1.5^{\circ}$, which is almost equivalent to the measurement error. The agreement between the forearm AOR and UIC might have clinical usefulness as a guide for the ulnar insertion point during IOM reconstruction or an anatomical reference point during the restoration of forearm bones and radial head fractures. We figured out that the proximal margin of the $\mathrm{CB}$ almost coincides with the RIC's apex, and the rest of the $\mathrm{CB}$ footprint extends from it to the distal slop of the RIC on the radial side. In addition, the anatomical consistency investigation for the location of each apex on the RIC and UIC showed that it appeared at a specific ratio to the total length of the radius and ulna, and there was no statistically significant difference between the right and left forearms. Marcotte and Osterman [3] reported that the ulnar insertion point is located at $33 \%$ of the ulnar length from the styloid process and that the radial insertion is located $60 \%$ of the radial length from the radial styloid process. Noda et al. [1] reported that the most distal and proximal ends of the radial origin of the $\mathrm{CB}$ were $53 \%$ and $64 \%$ of the total radial length from the tip of the radial styloid, whereas those of the ulnar insertion were $29 \%$ and $44 \%$ of the total ulnar length from the ulnar head. When we measured the position of each apex of the interosseous crests using a similar method, the results were consistent with those of these previous studies. The result of this study was that the RIC's apex, which the proximal margin of the $\mathrm{CB}$ coincides with, is about $40 \%$ distal from the top of the radial head. Such findings suggest the anatomical constancy of the topography of the interosseous crests. The observation that both ends of the $\mathrm{CB}$ commonly attach to the downslopes of the RIC and UIC will also provide a crucial biomechanical interpretation for understanding forearm rotation. As described by Chao and Morrey [5], forearm rotation follows a conical track with the ver- 
tex at the elbow, the base at the wrist, and the central axis as the AOR, which passes through the fovea of the radial head and the ulnar styloid base. If we look at the spatial configuration of the RIC and UIC from the perspective of the forearm circumduction cone, focusing on the angle of about $15^{\circ}$ formed by these two interosseous crests, the UIC's distal slope coincides with the forearm AOR, so it would be the height of this cone, and the RIC's distal slope would move along the slant around it. Therefore, the imaginary cone with a vertex angle of $30^{\circ}\left(15^{\circ} \times 2\right)$ drawn by these two interosseus crests during forearm rotation can be said to be a solid geometric core of the forearm circumduction. In addition to this characteristic motion trajectory of the forearm rotation, it is important to consider that the radius revolves around the ulna, and, at the same time, the axis of the radius rotates in proportion to the revolution angle. In this case of a complex rotatory motion between two long bones in the conical track constrained longitudinally by a broad, non-elastic band like the $\mathrm{CB}$, at the endpoint of rotation, this band structure could become distorted or wound around the shafts of both bones. As a result, disproportional tension in the band constraint would lead to restriction of the forearm rotation [9]. Therefore, some device would be required to offset the conical track of forearm rotation and to overcome the discrepancy between the proximal and distal fibers in the $\mathrm{CB}$ at any angle of rotation. We demonstrate that this could be achieved by the downslopes of the interosseous crests tracing two small conical tracks in opposite directions to the conical track of the forearm rotation (Fig. 5). This interpretation suggests that the interosseous crests of the radius and ulna are not simple bone-insertion sites for the $\mathrm{CB}$ but also stop distortion of the $\mathrm{CB}$ modulating tension and shift of the working segment in the CB during forearm rotation $[19,20]$.

We sought to elucidate clues to the geometric and biomechanical inevitability of the attachment points on the interosseous crests of the radius and ulna. Therefore, in particular, we believe that this study could contribute to the understanding and development of clinical methodologies for determining its isometric points on the osseous ridges when reconstructing the IOM $[3,21,22]$. Still, the restrictions in the resources and methods, the small number of samples, the paralleled direct measurement from the cadavers and the indirect measurement from the volunteers, and the demarcation of the $\mathrm{CB}$ using the backlit method with inherent non-uniformity are noted limitations of this study. Anatomically, most RICs show a clear and conspicuous shape, making it easy to identify their apex. On the other hand, the UICs show a relatively vague and flat tendency, suggesting the possibility of inter-observer differences in their definition. We clearly state that this bias may have impaired normality by caus-

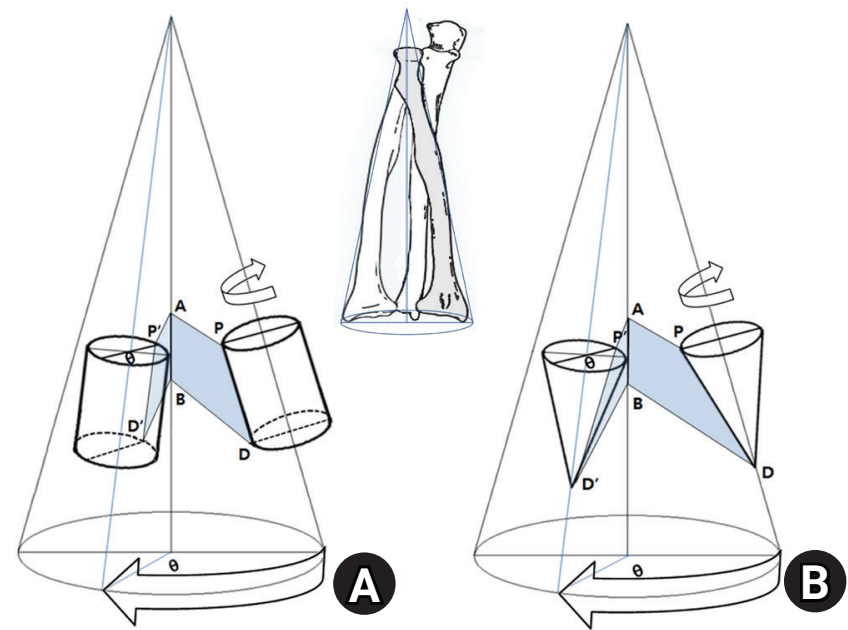

Fig. 5. Role of the longitudinal geometry of the interosseous crests of the radius and ulna. During rotation of the forearm categorized as circumduction, the radius is longitudinally constrained to the ulna by the central band (CB), a band-shaped ligament, and revolves around the forearm axis of rotation, tracing a conical track. One key consideration in this movement is that, when the forearm rotates a given angle, $\theta$ (revolution), the shaft of the radius also rotates by the same angle, $\theta$ (rotation). In this kinematics, if the interosseous crests where the $\mathrm{CB}$ inserts are modeled as cylinders (A), then, as the axis of the radius revolves and rotates along the conical track, distal fibers (line $\mathrm{BD}$ ) of the $\mathrm{CB}$ at the base would move across a longer diameter than proximal fibers (line AP) at the vertex. This disproportional rotation would result in greater tension in distal fibers as the forearm circumduction progresses, eventually restricting the rotation. However, suppose the $\mathrm{CB}$-insertion sites are modeled as slants of a cone in the opposite direction to the slant of the conical track of forearm circumduction (B). In this case, this unfavorable tension will not develop throughout the entire $\mathrm{CB}$, staying isometrically at any angle of forearm circumduction. This interpretation based on the topography might explain why the $\mathrm{CB}$ must necessarily insert into the distal downslopes of both interosseous crests.

ing irregularities in the ulnar side measurements, and we await follow-up studies using larger sample sizes and future validation of the methods used herein.

The UIC coincides with the AOR of the forearm, and the apex of the RIC coincides with the proximal CB footprint. Furthermore, the CB necessarily attaches to the downslope immediately distal to each apex of the interosseous crests of the radius and ulna, offsetting the disproportional conical track of the forearm circumduction to maintain the $\mathrm{CB}$ isometrically and isotonically in different degrees of forearm rotation.

\section{ORCID}

Suk-Hwan Jang

Kyung-Whan Kim

Hyo Seok Jang

Yeong-Seok Kim https://orcid.org/0000-0002-7880-2797 https://orcid.org/0000-0002-5233-3304 https://orcid.org/0000-0003-1235-8600 https://orcid.org/0000-0002-1599-2677 


\section{Hojin Kim \\ Youngbok Kim}

https://orcid.org/0000-0003-4489-9516

https://orcid.org/0000-0002-7966-1092

\section{REFERENCES}

1. Noda K, Goto A, Murase T, Sugamoto K, Yoshikawa H, Moritomo H. Interosseous membrane of the forearm: an anatomical study of ligament attachment locations. J Hand Surg Am 2009; 34:415-22.

2. Chandler JW, Stabile KJ, Pfaeffle HJ, Li ZM, Woo SL, Tomaino MM. Anatomic parameters for planning of interosseous ligament reconstruction using computer-assisted techniques. J Hand Surg Am 2003;28:111-6.

3. Marcotte AL, Osterman AL. Longitudinal radioulnar dissociation: identification and treatment of acute and chronic injuries. Hand Clin 2007;23:195-208.

4. Moritomo H, Noda K, Goto A, Murase T, Yoshikawa H, Sugamoto K. Interosseous membrane of the forearm: length change of ligaments during forearm rotation. J Hand Surg Am 2009; 34:685-91.

5. Chao EY, Morrey BF. Three-dimensional rotation of the elbow. J Biomech 1978;11:57-73.

6. Kapandji A. Biomechanics of pronation and supination of the forearm. Hand Clin 2001;17:111-22.

7. Adams JE. Forearm instability: anatomy, biomechanics, and treatment options. J Hand Surg Am 2017;42:47-52.

8. Hollister AM, Gellman H, Waters RL. The relationship of the interosseous membrane to the axis of rotation of the forearm. Clin Orthop Relat Res 1994;(298):272-6.

9. Soubeyrand M, Assabah B, Bégin M, Laemmel E, Dos Santos A, Crézé M. Pronation and supination of the hand: anatomy and biomechanics. Hand Surg Rehabil 2017;36:2-11.

10. LaStayo PC, Lee MJ. The forearm complex: anatomy, biomechanics and clinical considerations. J Hand Ther 2006;19:13744.

11. Mori K. Experimental study on rotation of the forearm--functional anatomy of the interosseous membrane. Nihon Seikeige- ka Gakkai Zasshi 1985;59:611-22.

12. Nakamura T, Yabe Y, Horiuchi Y. In vivo MR studies of dynamic changes in the interosseous membrane of the forearm during rotation. J Hand Surg Br 1999;24:245-8.

13. Nakamura T, Yabe Y, Horiuchi Y, Seki T, Yamazaki N. Normal kinematics of the interosseous membrane during forearm pronation-supination: a three-dimensional MRI study. Hand Surg 2000;5:1-10.

14. Hotchkiss RN, An KN, Sowa DT, Basta S, Weiland AJ. An anatomic and mechanical study of the interosseous membrane of the forearm: pathomechanics of proximal migration of the radius. J Hand Surg Am 1989;14(2 Pt 1):256-61.

15. Skahen JR 3rd, Palmer AK, Werner FW, Fortino MD. The interosseous membrane of the forearm: anatomy and function. J Hand Surg Am 1997;22:981-5.

16. Birkbeck DP, Failla JM, Hoshaw SJ, Fyhrie DP, Schaffler M. The interosseous membrane affects load distribution in the forearm. J Hand Surg Am 1997;22:975-80.

17. Poitevin LA. Anatomy and biomechanics of the interosseous membrane: its importance in the longitudinal stability of the forearm. Hand Clin 2001;17:97-110.

18. Forster RI, Sharkey NA, Szabo RM. Forearm interosseous ligament isometry. J Hand Surg Am 1999;24:538-45.

19. Manson TT, Pfaeffle HJ, Herdon JH, Tomaino MM, Fischer KJ. Forearm rotation alters interosseous ligament strain distribution. J Hand Surg Am 2000;25:1058-63.

20. Pfaeffle HJ, Fischer KJ, Srinivasa A, Manson T, Woo SL, Tomaino M. A model of stress and strain in the interosseous ligament of the forearm based on fiber network theory. J Biomech Eng 2006;128:725-32.

21. Adams JE, Culp RW, Osterman AL. Interosseous membrane reconstruction for the Essex-Lopresti injury. J Hand Surg Am 2010;35:129-36.

22. Meals CG, Forthman CL, Segalman KA. Suture-button reconstruction of the interosseous membrane. J Wrist Surg 2016; 5:179-83. 